\title{
Bioelectric Potential of Pothos under Light-Emitting Diode
}

\author{
Shin-ichi Shibata*, Takashi Oyabu ${ }^{1}$ and Haruhiko Kimura \\ Graduate School of Natural Science \& Technology, Kanazawa University, \\ Kakuma-machi, Kanazawa, Ishikawa 920-1192, Japan \\ ${ }^{1}$ Graduate School of Strategic Management, Kanazawa Seiryo University, \\ 10-1, Gosyo-machi, Kanazawa, Ishikawa 920-8620, Japan
}

(Received October 1, 2009; accepted April 26, 2010)

Key words: plant bioelectric potential, plant factory, LED, environmental sensing

Plant bioelectric potential changes according to environmental factors such as temperature, humidity, light intensity, and atmospheric pressure. Light is the fundamental factor for plant photosynthesis. In this paper, the relationship between bioelectric potential and the wavelength of emitted light (light-emitting diodes (LEDs): blue, green, red, and white) was examined. The bioelectric potential in darkness was adopted as a control and it was compared with those at various wavelengths. The bioelectric potential was measured as a function of time. Data were recorded every $0.1 \mathrm{~s}$ and summed over $1 \mathrm{~h}$. The summation value was adopted as a representative value. The correlation between the bioelectric potential under light emission and that of the control was determined. The correlation coefficient reached a maximum as the bioelectric potential under emission was shifted from $1-3 \mathrm{~h}$ of light exposure. The coefficient became lower as the wavelength of emitted light became higher. A coefficient of 0.84 under red light $(660 \mathrm{~nm})$ at a shifted time of $3 \mathrm{~h}$ was obtained, and 0.7 for green light $(525$ $\mathrm{nm})$ at a shifted time of $1 \mathrm{~h}, 0.5$ for blue $(475 \mathrm{~nm})$ without a shift. A coefficient of over 0.5 was obtained under white light at a shifted time of $1-2 \mathrm{~h}$. The obtained result contributes to our understanding of plant physiology. In addition, it contributes to the development of a plant environmental sensor and a plant factory.

\section{Introduction}

Food self-sufficiency is a problem in Japan and was about $40 \%$ in 2008. A significant amount of food is imported. This importation greatly increases the environmental load. It will become increasingly important to increase food self-sufficiency to an adequate level and ensure a steady supply of home-grown food. It is also necessary to reduce the use of agricultural chemicals and artificial fertilizers. Plant factories have received attention in recent years because the growth environment for a vegetable plant can be controlled to maintain a stable supply of food. ${ }^{(1)}$ The environment is not dependent on

${ }^{*}$ Corresponding author: e-mail: shin-ichi_shibata@blitz.ec.t.kanazawa-u.ac.jp 
the weather. These factories are pest-free; therefore, there is no need for pesticide use. As such, they can reduce adverse effect on the environment. The problem is that they are very cost-intensive.

The following environmental factors can be controlled to grow plants in a factory: temperature, light intensity, carbon dioxide concentration, and fertilizer amount. It is, however, difficult to maintain the optimum growth environment for plants because there is insufficient information concerning optimal environmental conditions. However, bioelectric potential constitutes a source of information from the plant. It is clear that the potential changes according to environmental factors, for example, light intensity. ${ }^{(2)}$ It is also reported that the growth of a plant differs with light wavelength. ${ }^{(3)}$ A study on the relationship between environmental factors and bioelectric potential has been planned. ${ }^{(4)}$

Plant bioelectric potential is studied, as are its changes with the wavelength of light emitted from light-emitting diodes (LEDs), as part of the development of a plant factory. Bioelectric potential can be used to determine the plant condition and production can also be increased if the plant growing environment could be evaluated on the basis of bioelectrical potential. The correlation between the control and the bioelectric potentials under red, green, and blue lights increases when each bioelectric potential is shifted for several hours to the control bioelectric potential, which is measured in darkness. Therefore, there is a considerable time lag in the bioelectric potential under LED lights. The bioelectric potential under red light is similar to that of the control. The wavelength of red light is longer than those of other lights. The correlation for the blue light is markedly reduced. The energy of blue light is higher than those of green and red lights. It is also higher than that of white light, which includes various light wavelengths. It is considered that these characteristics are strongly associated with vegetable physiology. It is considered that investigation of vegetable physiology will be stimulated by this study. Our findings will contribute to the development of a deeper understanding of plant physiology.

\section{Experimental Procedure}

It is said that life on this planet is under the influence of a life field, for example electromagnetic waves. ${ }^{(5)}$ Plants are also influenced transiently by this field, any environmental factors, and potential changes. The optimum environment for a plant can be provided by understanding plant bioelectric potential and its correlation with environmental conditions. Then, plants can be used as environmental sensors. However, it is difficult to carry out an experiment to determine the relationship between potential and environmental factors, for example, temperature and atmospheric pressure, where these factors are precisely controlled. This experiment was carried out in an indoor environment because the variation in environmental conditions there is relatively small. A pothos plant (Epipremnum aureum) was used in this study. The plant is familiar to humans. It was kept in a pot with an internal diameter of $10 \mathrm{~cm}$ and a height of $14 \mathrm{~cm}$. The plant height from the bottom of the pot was about $55 \mathrm{~cm}$. Three of these potted plants were prepared.

The plants were installed in an experimental chamber (inner volume: about $230 \mathrm{~L}$ ) 
and the experiment was carried out with opening the door to remove environmental factor affecting the chamber. The chamber is set up to block the light from outside. The measuring system (bioelectric sensor, temperature and humidity sensors, and atmospheric sensor) is incorporated into the chamber. The system is shown in Fig. 1. Two electrodes are attached to two leaves of the plant, which are near the surface of the pot soil and are adjacent. The ground electrode is connected to the soil. The electrode is composed of aluminum and its area is $3 \mathrm{~cm}^{2}$. The differential signal among the electrodes is amplified 100-fold (KEYENCE Co., OP-3009). Then, the signal is input into a portable computer through an A-D converter (KEYENCE Co., NR-350). The sampling interval is 0.1 s. The LED panel (LED unit: CCS Inc., ISL-150×150) is set up at a height of $90 \mathrm{~cm}$ from the chamber floor, and the LED controller (CCS Inc., ISC-201-2) is set outside the chamber. Photographs of the experimental arrangement and LED unit are shown in Fig. 2. Figure 2(a) shows the chamber in which a plant is installed and Fig. 2(b) shows the LED panel. There are four types of LED panels, namely, white, blue, green, and red light units. The white unit emits light of various frequencies. The performances of all units are summarized in Table 1. Effective photosynthetic flux density is constant at 100 $\mu \mathrm{E}$ and the value is 100 . The illuminance (lux) for each color is changed from 394 (red) to 4775 lux (green). The irradiances $\left(\mathrm{w} / \mathrm{m}^{2}\right)$ are also summarized in the table. The data were measured at $10 \mathrm{~cm}$ from the light source. Photosynthetic flux density affects plant photosynthesis.

\section{Results and Discussions}

\subsection{Original data and the summation characteristic}

It is considered that various types of information are provided by the plant bioelectric potential. Plants react to temperature, humidity, atmospheric pressure, light intensity, and carbon dioxide concentration changes instantaneously or over the long term. The widespread use of plants should increase markedly when their response characteristics are examined and publicized. A potted foliage plant, pothos, was used in this study and

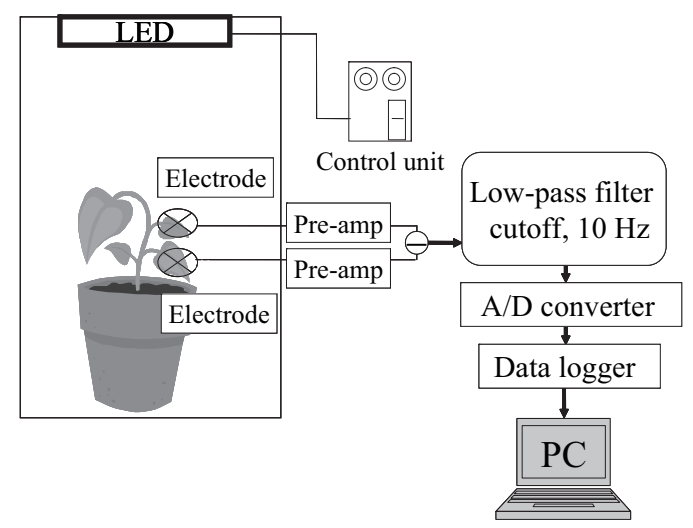

Fig. 1. Schematic diagram of the experimental system. 


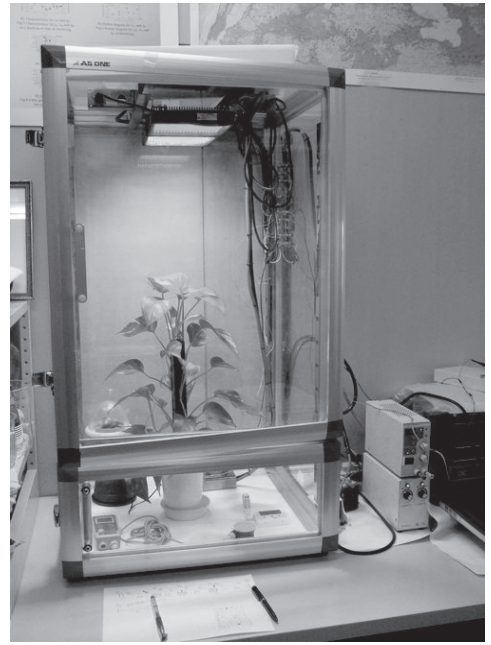

(a)

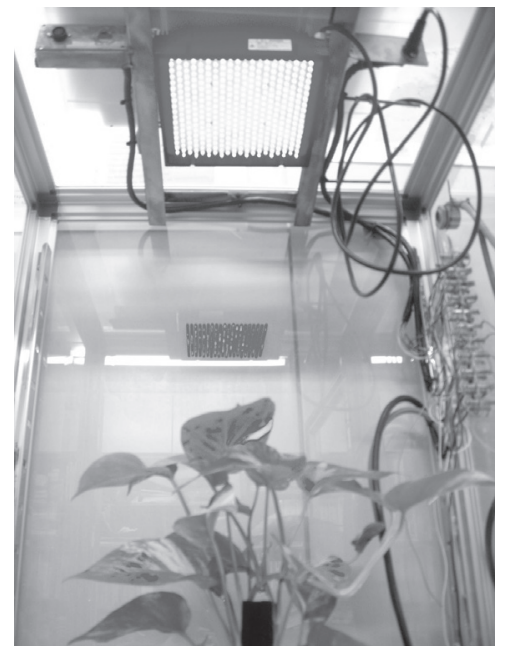

(b)

Fig. 2. Photographs of the experimental chamber and the LED unit. The subjective plant is installed in the chamber. (a) Experimental chamber. (b) LED unit.

Table 1

Performance of LED units.

\begin{tabular}{lcrcc}
\hline & $\mu \mathrm{E}$ & $\mathrm{lux}$ & $\mathrm{w} / \mathrm{m}^{2}$ & $\mathrm{~nm}$ \\
\hline White & 100 & 2835 & 23 & - \\
Blue & 100 & 929 & 18 & 475 \\
Green & 100 & 4755 & 21 & 525 \\
Red & 100 & 394 & 25 & 660 \\
\hline
\end{tabular}

Measured distance: $100 \mathrm{~mm}$

the experiment was carried out in an indoor environment. Temperature and humidity do not change significantly indoors. ${ }^{(6)}$ Therefore, the transient changes could not be detected in a room. It is expected that the responses to the environmental factors are medium- and long-term changes such as seasonal effects. It would appear that measured bioelectric potential is a response due to wavelength under such environmental conditions. Consequently, the bioelectric potential change with light wavelength can be investigated. The parameter is wavelength, namely, those of blue, green, red, and white light.

The measured bioelectric potentials, which are original data, are shown in Fig. 3. The upper panel shows the bioelectric potentials in darkness. The lower panel shows that under red light. The data is input every $0.1 \mathrm{~s}$. Each bioelectric potential has 600 data values, namely, they represent the bioelectric potentials for $1 \mathrm{~min}$. It is difficult to observe a trend in the bioelectric potentials shown in Fig. 3. It is considered that the 


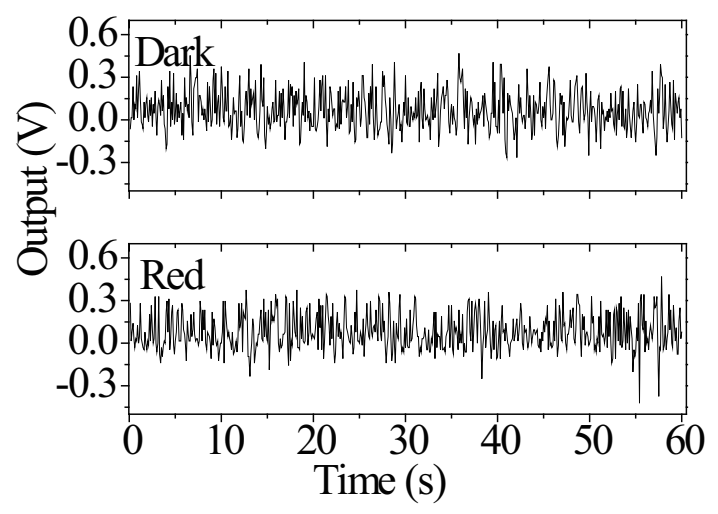

Fig. 3. Original characteristic of pothos bioelectric potential in darkness and under red light.

environmental factors change moderately in a room. Therefore, the summation values $\left(v_{\mathrm{h} 1}, v_{\mathrm{m} 30}\right)$ of the absolute input data for $1 \mathrm{~h}$ and $30 \mathrm{~min}$ are adopted to observe a trend in the potential. $v_{\mathrm{h} 1}$ (absolute summation value for $1 \mathrm{~h}$ ) is used in this experiment, and the value is the summation of 36,000 input data values. An example of $v_{\mathrm{h} 1}$ during nighttime (18:00-08:00) is shown in Fig. 4. The bioelectric potentials under red light and in darkness are shown in the figure. The value obtained in darkness is higher than that obtained under red light. The values obtained under blue and green lights are also lower than that obtained in darkness. It is considered that the plant is stressed in darkness. The same feature is obtained at a temperature lower than $10^{\circ} \mathrm{C} .(7)$ The characteristics for temperature, humidity, and atmospheric pressure in the experiment are also shown in Fig. 5. The characteristics during night-time (23:00-05:00) are focused on. The fluctuations in temperature and humidity during night-times are lower than those during daytime. The fluctuation ranges for the factors are $18-20^{\circ} \mathrm{C}, 35-40 \% \mathrm{RH}$, and $1,000-1,005 \mathrm{hPa}$ in the figure.

\subsection{Potential under different light frequencies}

It is important to understand the characteristics of the plant bioelectric potential change under four kinds of LED lights. The bioelectric potential $\left(v_{\mathrm{h} 1}\right)$ in darkness is adopted as a control in this experiment. It is desired that the features under each light wavelength can be derived from the experimental results. It is also considered that the plant cannot respond rapidly to a wavelength and rather responds in later stages, as shown by the results of a preliminary experiment. The bioelectric potential response shows a tendency of more than a couple of hours delay or speeding up compared with those in darkness. The responses of the bioelectric potentials under the three lights (blue, green, and red) are more or less several hours slower or faster (per $1 \mathrm{~h}$ ) than that in darkness. It is believed that each light wavelength affects growth of each part of the plant and disrupts the circadian rhythm. The correlation coefficient $(\gamma)$ between the shifted bioelectric potential (under lights) and control bioelectric potential (darkness) is 


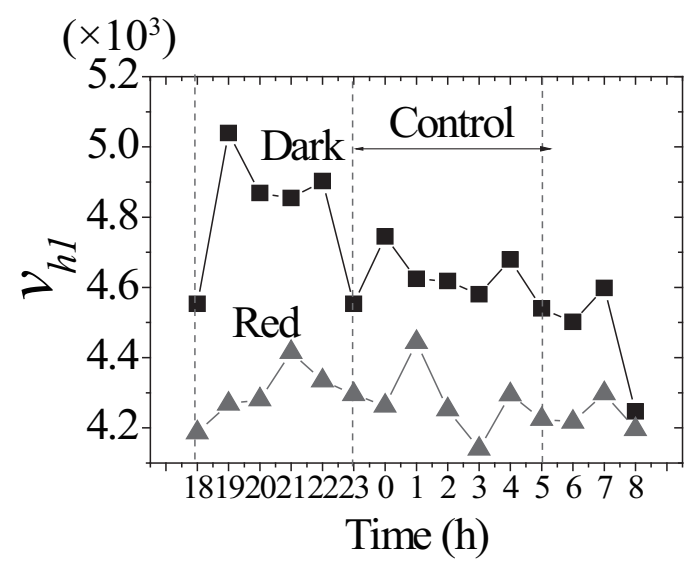

Fig. 4. Summation characteristic $v_{\mathrm{h} 1}$ for original bioelectric potential in darkness and under red light during night-time hours.

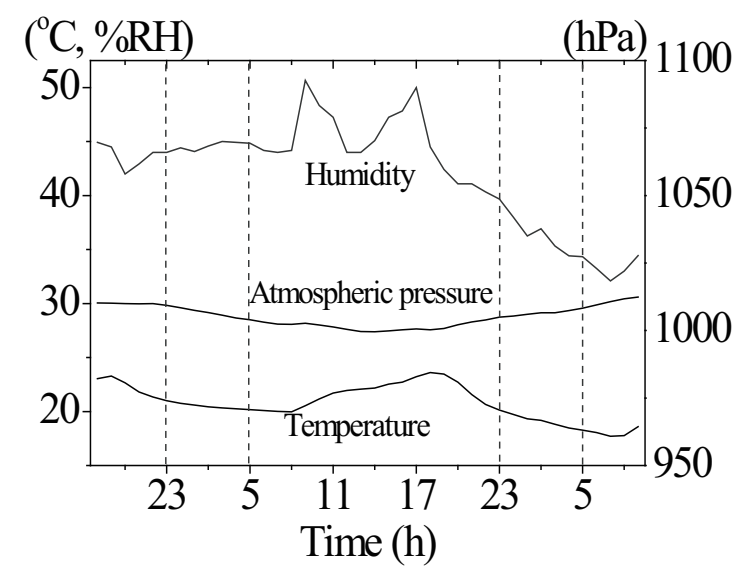

Fig. 5. Characteristics of temperature, humidity, and atmospheric pressure during night-time.

introduced. The results are shown in Fig. 6. Figures 6(a)-6(c) show similar trends. The bioelectric potentials for blue and green lights particularly resemble each other. The shifted period that exhibits the maximum correlation $\left(\gamma_{\max }\right)$ differs with wavelength. $\gamma_{\max }$ has the maximum value at the shifted period of zero (no shift) under blue light. It has the maximum value at the shifted period of $1 \mathrm{~h}$ under green light and at the period of $3 \mathrm{~h}$ under red light.

The scatter diagrams for the control $\left(v_{\mathrm{hl} 1 \text {-dark }}\right)$ and each bioelectric potential under three LED units are shown in Fig. 7. Figure 7(a) is for blue light, Fig. 7(b) is for green light, and Fig. 7(c) is for red light. The correlation coefficient of the scatter diagram for the three LED units shows the trend that the coefficient increases as the LED wavelength 


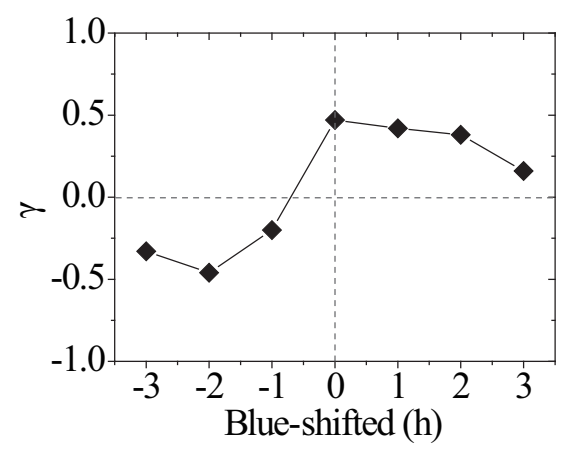

(a)

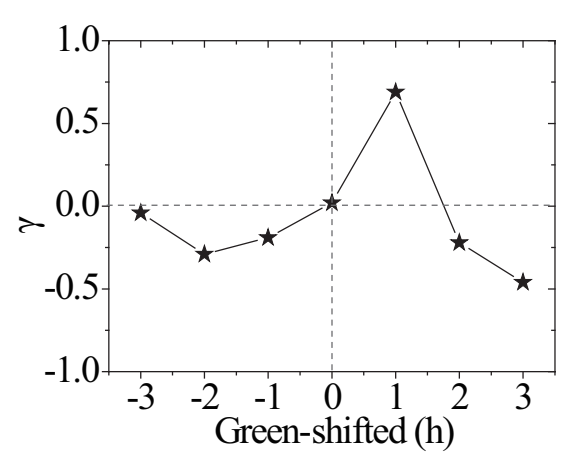

(b)

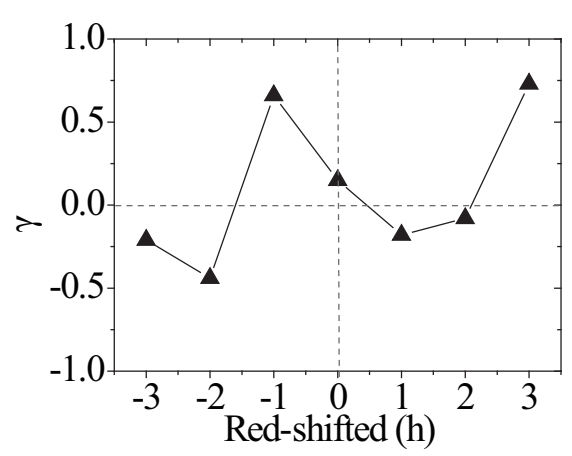

(c)

Fig. 6. Relationship between correlation coefficient and shifted time for three lights. Characteristic for blue light. (b) Characteristic for green light. (c) Characteristic for red light.

decreases. The correlation coefficient is exhibited in Fig. 8. In the red LED experiment, the $\gamma_{\max }$ has a maximum value of 0.84 at the shifted period of $3 \mathrm{~h}$.

\section{3 $\mathrm{v}_{\text {hl }}$ histogram for light from three LED units}

It is considered that blue and red lights contribute to the photosynthesis of vegetable plants. ${ }^{(8,9)}$ In photosynthesis, chlorophyll, which absorbs light, has a fundamental role. Chlorophyll mainly absorbs light of red and blue wavelengths. The mechanism involved remains only partly understood, and experimental studies are required. The fluctuation of bioelectric potential with light wavelength is examined in this experiment. The potential is affected by various types of environmental factors. It is difficult to understand the features from the original signal. The summation value $\left(v_{\mathrm{h} 1}\right)$ for $1 \mathrm{~h}$ is adopted. The histogram of $v_{\mathrm{h} 1}$ is introduced and analyzed in this section and the result is shown in Fig. 9. Figure 9(a) is for blue light, Fig. 9(b) is for green light, and Fig. 9(c) is for red light. There is an observed trend that average $v_{\mathrm{h} 1}$ decreased as light wavelength decreased, 


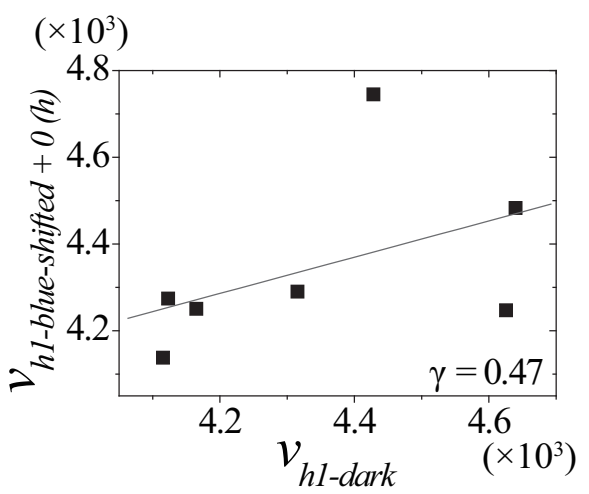

(a)

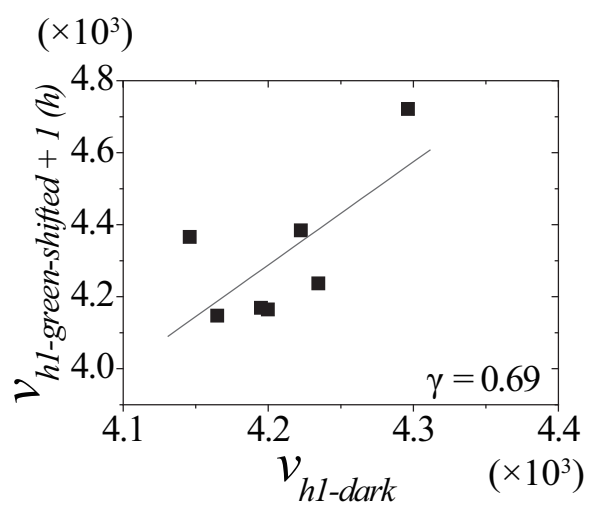

(b)

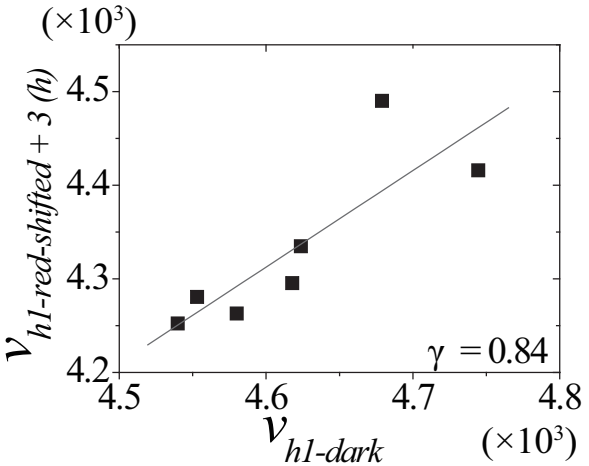

(c)

Fig. 7. Scatter diagram between $v_{\mathrm{h} 1}$ and $v_{\mathrm{hl} \text {-dark }}$ under each LED unit when the correlation coefficient takes a maximum value. (a) Characteristic for blue light. (b) Characteristic for green light. (c) Characteristic for red light.

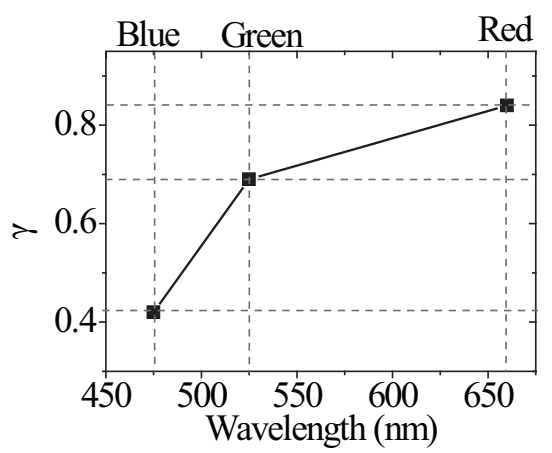

Fig. 8. Maximum correlation coefficient as a function of light wavelength. 


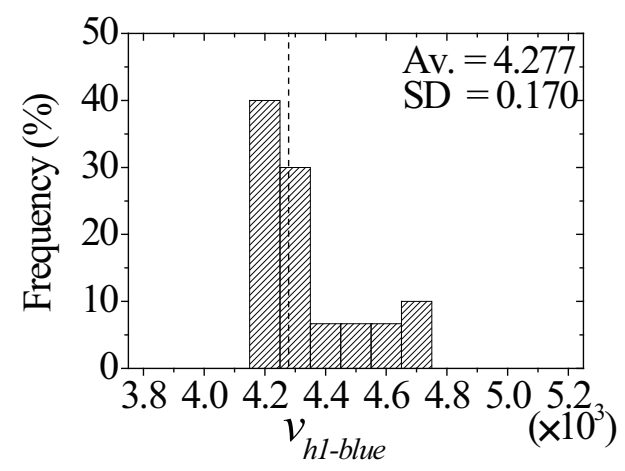

(a)

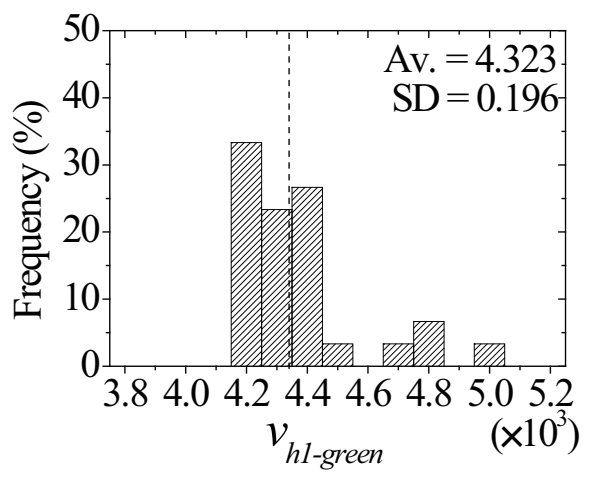

(b)

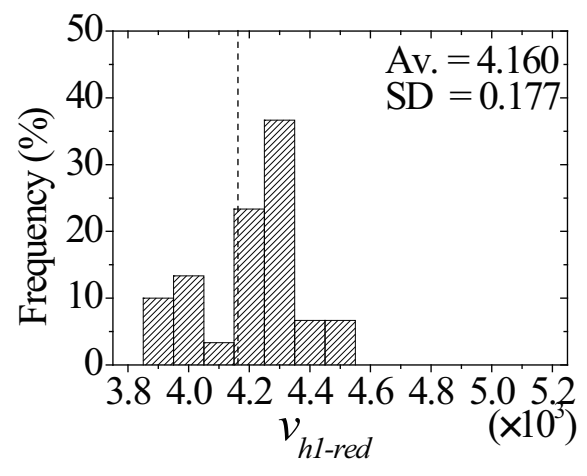

(c)

Fig. 9. Histogram of $v_{\mathrm{h} 1}$ for each LED unit. (a) Histogram for blue light. (b) Histogram for green light. (c) Histogram for red light.

but the value for green light is slightly higher than that for blue light. The relationship between light wavelength and average $v_{\mathrm{h} 1}$ is shown in Fig. 10.

\subsection{Bioelectric potential under white LED}

Bioelectric potential under direct light was examined in the previous section. In this section, the potential under white light, which has various frequencies, is examined. The plot in Fig. 8 is for white light. The correlation coefficient $(r)$ between the control (in darkness) and $v_{\mathrm{h} 1}$ under white light is shown in Fig. 11. The $v_{\mathrm{h} 1}$ under white light is shifted from -3 and to $+3 \mathrm{~h}$ with respect to that of the control. The maximum correlation coefficient $\left(\gamma_{\max }\right)$ of 0.76 is derived at the shifted time of $+2 \mathrm{~h}$. The graph of correlation coefficient shown in Fig. 11 is similar to that shown in Fig. 6(b). A histogram of $v_{\mathrm{h} 1}$ under white light is prepared. The average $v_{\mathrm{h} 1}$ is 4337 and the value is higher than those 


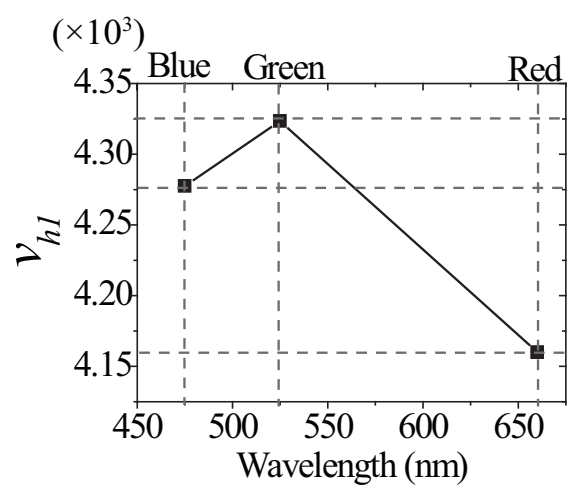

Fig. 10. Average $v_{\mathrm{h} 1}$ as a function of wavelength of LED unit.

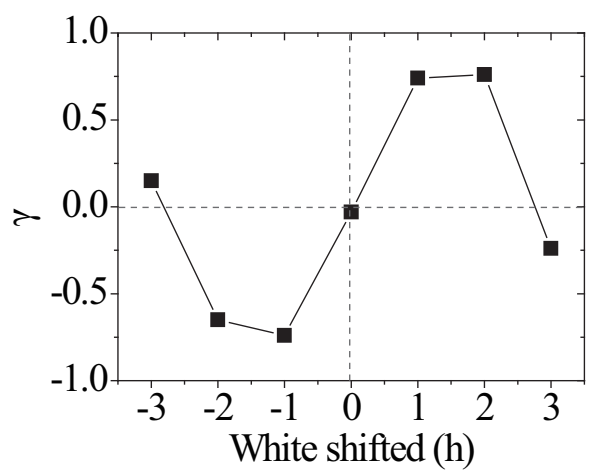

Fig. 11. Correlation coefficient as a function of shifted time under white LED.

under blue, green, and red lights. It is considered that the average under white light becomes higher owing to the various types of frequencies. White light exhibits various frequencies. Each part of the plant grows under various light frequencies. Consequently, it is considered that $v_{\mathrm{h} 1}$ becomes higher under white light.

White light, which is similar to sunlight, plays an important role in plants. The characteristics of $v_{\mathrm{h} 1}$ under white light for two days are shown in Fig. 12. The $v_{\mathrm{h} 1}$ for day one is designated as "White1" and that for day two, "White2" in the figure. The two characteristics are similar but the widths of fluctuation and times are slightly different. The circadian rhythm of the plant is disturbed by the continuous exposure to light for $24 \mathrm{~h}$.

\section{Conclusions}

There have been few studies of plant bioelectric potential, despite the importance of plants to humans. In general, many studies of plants are carried out from various viewpoints and a wide range of applications for plants has been accomplished. This experiment was carried out during night-time and indoors. The environmental factors 


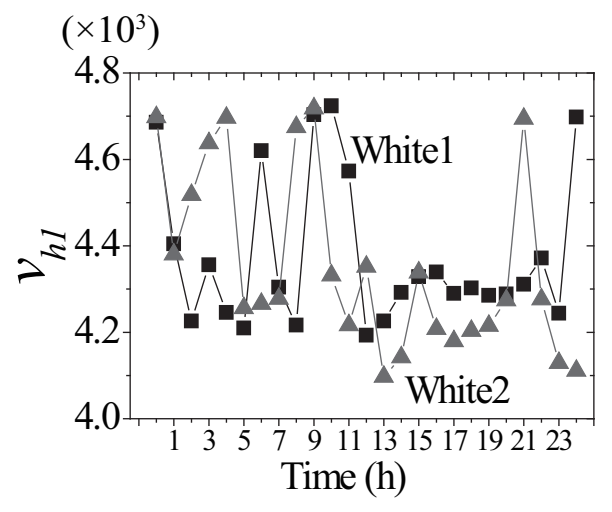

Fig. 12. Summation characteristic $v_{\mathrm{h} 1}$ under white LED for $24 \mathrm{~h}$.

do not fluctuate largely in these circumstances. The goal of the study was to determine the trend of $v_{\mathrm{h} 1}$ under LED units. The experimental results denoted the same tendency of $v_{\mathrm{h} 1}$ under similar conditions. The $v_{\mathrm{h} 1}$ fluctuates with light wavelength. The experiments were carried out under two conditions. One was darkness and the other was light. The bioelectric potential in darkness was adopted as the control and compared with those under four types of lights, namely, blue, green, red, and white. The control bioelectric potential is adopted as the base and the bioelectric potentials under four lights are shifted. The correlation coefficient between the two bioelectric potentials is derived. It is clear that the maximum correlation coefficient $\left(\gamma_{\max }\right)$ increases as light wavelength increases. $v_{\mathrm{h} 1}$ becomes smaller as wavelength increases. $v_{\mathrm{h} 1}$ under the white light becomes larger than those under other lights. The result is dependent on vegetable physiology. Study of the relationship between plant bioelectric potential and vegetable physiology will be carried out and a deeper understanding of this will gradually be developed.

\section{References}

1 M. Takatsuji: Introduction to Plant Factories and Examples (Syoukabou, Tokyo, 2007) (in Japanese).

2 T. Shimbo, H. Kimura and T. Oyabu: Soc. Envir. Instrum. Control Automation Jpn. 13 (2008) 27.

3 K. Atushi: Plants Offer the Future (Kyoritsu-syupan, Tokyo, 2002) (in Japanese).

4 H. Nanbo, S. Kitajima, T. Shimbo, H. Kimura and T. Oyabu: Soc. Plant Engin. Jpn. 20 (2008) 18.

5 H. S. Burr: Blueprint for Immortality (Nihon kyoubun, Tokyo, 2006).

6 T. Shimbo, M. Fuji, A. Sawada, T. Oyabu and H. Kimura: IEEJ Trans. SM 127 (2007) 425.

7 T. Shimbo, T. Oyabu, Y. Hasegawa, S. Hirobayashi and K. Takenaka: IEEJ Trans. SM 125 (2005) 253.

8 N. Kasai: Plants Turn the Earth (Kagaku Dojin, Kyoto, 2007) (in Japanese).

9 T. Oyabu and T. Katube: Plant Bioelectric Potential and Its Communication (Kaibundo, Tokyo, 2009) (in Japanese). 